\title{
A Dispersive Optical Model Analysis of the Protons Scattering by Titanium Element Nucleus and Its Natural Isotopes
}

\author{
Haiddar Al-Mustafa, Anees Belal \\ Department of Physics, Faculty of Science, Al-Baath University, Homs, Syria \\ Email address: \\ HAIDDAR2018@gmail.com (H. Al-Mustafa), dr anees blal $a$ gmail.com (A. Belal)
}

\section{To cite this article:}

Haiddar Al-Mustafa, Anees Belal. A Dispersive Optical Model Analysis of the Protons Scattering by Titanium Element Nucleus and Its Natural Isotopes. Nuclear Science. Vol. 4, No. 4, 2019, pp. 44-51. doi: 10.11648/j.ns.20190404.12

Received: November 10, 2019; Accepted: November 12, 2019; Published: December 10, 2019

\begin{abstract}
A dispersive optical model analysis of the proton scattering by titanium element nucleus and its natural isotopes is applied to the construction of the complex single-particle mean field starting from Fermi energy value to the energy value $100 \mathrm{MeV}$ and for constant input values of the parameters of this mean field. This mean field is called (coulomb-nuclear) interference potential, that contains (spin-orbit) coulomb term. The results according to DOMACNIP program that has been designed for that purpose would contain: continuous energy variation of the depths of the real and imaginary parts of the mean field, which are connected by dispersion relations were compared with these resulting from global parameterization of the optical model potential. In addition to continuous energy variation of the real radius parameter of the Wood-Saxon approximation to the mean field potential with its Hatree-Fock approximation of the nonlocal potential. Consequently, our results for the continuous energy variations of the predicted total reaction cross section within the energy range (1-100) $\mathrm{MeV}$, and with calculation step of the pervious range whose magnitude $(1 \mathrm{MeV})$, differential cross sections, Ratio of the differential elastic scattering cross section to Rutherford cross section, polarization for selected energy showed the excellent agreement with available experimental data and with these resulted from global parameterization of the optical model potential.
\end{abstract}

Keywords: Dispersive Optical Model Analysis (DOMA), (Coulomb-Nuclear) Interference Potential (CNIP), Dispersion Relations (DR), Mean Field, Fermi Energy, Cross Section, Polarization

\section{Introduction}

The nuclear optical model potential describes the motion of one nucleon, bound or unbound, in the mean field of all the other nucleons comprising the nucleus. The field due to the sum of all the individual nucleon-nucleon interactions is thus represented by a simple one-body potential. This approximation greatly simplifies the calculation of a wide range of nuclear structure and nuclear reaction phenomena, in addition to the excellent agreement with experimental data (1).

The phenomenological optical model potential for nucleonnucleus scattering, $U$, is defined as [2-6]:

$$
U(r, E)=-V_{V}(r, E)-V_{S O}(r, E) \cdot \vec{\sigma} \cdot \vec{l}+V_{S}(r, E)+V_{C}(r)+i\left(-W_{V}(r, E)-W_{S}(r, E)+W_{S O}(r, E) \cdot \vec{\sigma} \cdot \vec{l}\right)
$$

Where $V_{V, S}$ and $W_{V, S, S O}$ are the real and imaginary components of the volume-central $(V)$, surface-central $(S)$ and spin-orbit $(S O)$ potentials, respectively. $E$ is the LAB energy of the incident particle in $\mathrm{MeV}$. All components are separated in energy-dependent well depths, $V_{V}, V_{S}, W_{V}, W_{S}$ and $W_{S O}$, and energy-independent radial parts $f$, namely

$$
\begin{gathered}
V_{V}(r, E)=V_{V}(E) f\left(r, R_{V}, a_{V}\right) \\
W_{V}(r, E)=W_{V}(E) f\left(r, R_{V}, a_{V}\right) \\
W_{S}(r, E)=-4 a_{S} W_{S}(E) \frac{d}{d r} f\left(r, R_{S}, a_{S}\right) \\
V_{S}(r, E)=-4 a_{S} V_{S}(E) \frac{d}{d r} f\left(r, R_{S}, a_{S}\right) \\
V_{S O}(r, E)=V_{S O}(E)\left(\frac{\hbar}{m_{\pi} c}\right)^{2} \frac{1 d}{r d r} f\left(r, R_{S O}, a_{S O}\right) \\
W_{S O}(r, E)=W_{S O}(E)\left(\frac{\hbar}{m_{\pi} c}\right)^{2} \frac{1 d}{r d r} f\left(r, R_{S O}, a_{S O}\right)
\end{gathered}
$$


The form factor $f\left(r, R_{i}, a_{i}\right)$ is a Wood-Saxon shape

$$
f\left(r, R_{i}, a_{i}\right)=\frac{1}{\left[1+e^{\left(\frac{r-R_{i}}{a_{i}}\right)}\right]}
$$

Where the geometry parameters are the radius $R_{i}=r_{i} A^{\frac{1}{3}}$, with $A$ the atomic mass number, and the diffuseness parameters $a_{i}, i=V, S O, S$. For charged particles, the coulomb term $V_{C}$, as usual, is given by that of a uniformly charged sphere

$$
\begin{gathered}
V_{C}(r)=\left(Z Z^{\prime} e^{2} / 2 R_{C}\right)\left(3-r^{2} / R_{C}^{2}\right): r \leq R_{C} \\
=Z Z^{\prime} e^{2} / r: r \geq R_{C}
\end{gathered}
$$

With $Z\left(Z^{\prime}\right)$ the charge of the target (projectile), and $R_{C}=r_{C} A^{\frac{1}{3}}$ the Coulomb radius.

In addition of the term $U_{C, S O}$, the Coulomb spin-orbit term that is assumed to have the form:

$$
U_{C, S O}=\left(\mu_{P}-\frac{1}{2}\right) \frac{1}{M_{p}^{2} c^{2}}\left[\frac{1}{r} \frac{d}{d r} V_{C}(r)\right] \vec{\sigma} \cdot \vec{l}
$$

Where $\mu_{P}$ is the proton magnetic moment in nuclear magnetons.

We have got the (Coulomb-Nuclear) interference potential which is expressed as follows:

$$
U_{C N I P}(r, E)=V_{o p t}(r, E)+i W_{o p t}(r, E)
$$

Where:

$$
\left\{\begin{array}{c}
V_{o p t}(r, E)=U_{C}(r)+U_{S}(r, E)-U_{V}(r, E)+ \\
\left\{-U_{S O}(r, E)+U_{C, S O}(r)\right\}\left(\begin{array}{c}
l \\
o r \\
-l-1
\end{array}\right) \\
W_{o p t}(r, E)=-W_{V}(r, E)-W_{S}(r, E)+W_{S O}(r, E)\left(\begin{array}{c}
l \\
o r \\
-l-1
\end{array}\right)
\end{array}\right.
$$

Here, $l$ for $j=l+1 / 2$ and $-l-1$ for $j=l-1 / 2$

By solving the Schrödinger equation numerically with this complex potential yields a wealth of valuable information; it returns a prediction for the basic observables, namely the elastic angular distribution and polarization, the reaction and total cross section, and the detailed information of the calculation methodology that is showed in the reference [7].

The essential value of a good optical model is that it can reliably predict these quantities for energies and nuclides for which no measurements exist. Also, the quality of the not directly observable quantities that are provided by the optical model has an equally important impact on the evaluation of the various reaction channels.

The dispersive optical model analysis describes the continuous energy variation of the nuclear mean field potential components depths and connection between the real parts and imaginary parts of the mean field by a dispersion relation, and so the reliable determination of the mean field is perfect by comparing a prediction of the cross sections with these are measured experimentally.

These dispersion relations are a natural result of the causality principle that a scattered wave cannot be emitted before the arrival of the incident wave. The dispersion component stems directly from the absorptive part of the potential,

$$
\Delta \mathcal{V}(r, E)=\frac{\mathcal{P}}{\pi} \int_{-\infty}^{+\infty} \frac{\mathcal{W}\left(r, E^{\prime}\right)}{E^{\prime}-E} d E^{\prime}
$$

Where $\mathcal{P}$ denotes the principal value. The total real central potential can be written as the sum of a Hatree-Fock term $\mathcal{V}_{H F}(r, E)$ and the total dispersion potential $\Delta \mathcal{V}(r, E)$

$$
\mathcal{V}(r, E)=\mathcal{V}_{H F}(r, E)+\Delta \mathcal{V}(r, E)
$$

Since $\mathcal{W}(r, E)$ has a volume and a surface component, the dispersive addition is,

$$
\begin{gathered}
\Delta \mathcal{V}(r, E)=\Delta \mathcal{V}_{V}(r, E)+\Delta \mathcal{V}_{D}(r, E) \\
=\Delta V_{V}(E) f\left(r, R_{V}, a_{V}\right)-4 a_{D} \Delta V_{D}(E) \frac{d}{d r} f\left(r, R_{D}, a_{D}\right)
\end{gathered}
$$

Where the volume dispersion term is given by

$$
\Delta V_{V}(E)=\frac{\mathcal{P}}{\pi} \int_{-\infty}^{+\infty} \frac{W_{V}\left(E^{\prime}\right)}{E^{\prime}-E} d E^{\prime}
$$

And the surface dispersion term is given by

$$
\Delta V_{D}(E)=\frac{\mathcal{P}}{\pi} \int_{-\infty}^{+\infty} \frac{W_{D}\left(E^{\prime}\right)}{E^{\prime}-E} d E^{\prime}
$$

In general, "(10)" \& “(11)" cannot be solved analytically. However, under certain plausible conditions, analytical solutions exist. Under the assumption that the imaginary potential is symmetric with respect to the Fermi energy $E_{F}$

$$
W\left(E_{F}-E\right)=W\left(E_{F}+E\right)
$$

Where $W$ denotes either the volume or surface term, we can rewrite the dispersion relation as,

$$
\Delta V(E)=\frac{2}{\pi}\left(E-E_{F}\right) \mathcal{P} \int_{E_{F}}^{\infty} \frac{W\left(E^{\prime}\right)}{\left(E^{\prime}-E_{F}\right)^{2}-\left(E-E_{F}\right)^{2}} d E^{\prime}
$$

There are many published studies for detailed analyses of data for the scattering state by using dispersion relations. Some of these studies that started from the energy variation of the moments of the imaginary potential have used BrownRho expression and assumed that the Hatree-Fock field has the linear energy dependence.

The present paper aims at presenting the dispersive optical model analysis (DOMA) of the protons scattering by titanium element nucleus and its natural isotopes and comparing the results with these resulted from global parametrization of the optical model potential and averrable experimental data within energy range (1-100) $\mathrm{MeV}$ and with calculation step of the previous range whose magnitude $1 \mathrm{MeV}$.

\section{Methodology}

The dispersive optical model analysis is summarized as follows [2-4, 8]:

\subsection{Volume Integral per Nucleon}

Determining the continuous energy variation of the volume integral per nucleon by using Brown-Rho expression: 
For the central imaginary part of the nuclear mean field:

$$
J_{W}(E)=\beta \frac{\left(E-E_{0}\right)^{2}}{\left(E-E_{0}\right)^{2}+\rho_{W}^{2}}
$$

The imaginary part has a volume and a surface component, the volume component is,

$$
J_{W_{V}}(E)=\beta \frac{\left(E-E_{0}\right)^{2}}{\left(E-E_{0}\right)^{2}+\rho_{W_{V}}^{2}}
$$

So, the surface component is,

$$
J_{W_{S}}(E)=J_{W}(E)-J_{W_{V}}(E)
$$

where $\beta, \rho_{W}, \rho_{W_{V}}$ denote Brown-Rho parameters, $E_{0}$ is:

$$
E_{0}=\frac{E_{F}}{2}
$$

Where, $E_{F}$, the Fermi energy in $\mathrm{MeV}$, that is defined as the energy halfway between the last occupied and the first unoccupied shell of the nucleus, determined from the experimental masses as follows:

$$
\begin{gathered}
E_{F}=\frac{E_{F}^{+}+E_{F}^{-}}{2} \\
E_{F}^{+}=M_{A+1}-M_{A}-m \\
E_{F}^{-}=M_{A}-M_{A-1}-m
\end{gathered}
$$

Where $E_{F}^{+}$is the negative of the separation energy of a nucleon from the $(\mathrm{A}+1)$-nucleon system. Also, $E_{F}^{-}$is the negative of the separation energy of a nucleon from the Anucleon system, $m$ is the atomic mass of the incident particle.

\subsection{Depths of the Volume and Surface Absorption of the Mean Field}

Determining the continuous energy variation of the volume and surface absorption depths,

$$
\begin{gathered}
\mathrm{W}_{\mathrm{V}}(\mathrm{E})=\mathrm{J}_{\mathrm{W}_{\mathrm{V}}}(\mathrm{E}) / \mathrm{g}_{\mathrm{wv}}, \mathrm{MeV} \\
\mathrm{W}_{\mathrm{S}}(\mathrm{E})=\mathrm{J}_{\mathrm{W}_{\mathrm{S}}}(\mathrm{E}) / \mathrm{g}_{\mathrm{ws}}, \mathrm{MeV}
\end{gathered}
$$

Where $g_{w v}, g_{w s}$ can be written as follows:

$$
\begin{gathered}
g_{w \mathrm{v}}=\frac{4 \pi}{3} \frac{R_{w \mathrm{v}}^{3}}{A_{t} * A_{p}}\left[1+\left(\frac{\pi a_{w \mathrm{v}}}{R_{w \mathrm{v}}}\right)^{2}\right] \\
g_{w \mathrm{~s}}=\frac{16 \pi R_{w s}^{2} a_{w s}}{A_{t^{*}} A_{p}}\left[1+\frac{1}{3}\left(\frac{\pi a_{w s}}{R_{w s}}\right)^{2}\right]
\end{gathered}
$$

Where $R_{w v}=r_{w v} A_{t}^{\frac{1}{3}}, a_{w v}, R_{w s}=r_{w s} A_{t}^{\frac{1}{3}}, a_{w s}$ are the radius and diffuseness parameters of the volume and surface absorption.

\subsection{Volume Integral per Nucleon of Dispersive Corrections of the Real Part of the Mean Field}

Determining the continuous energy variation of the volume integral per nucleon of dispersive corrections of the real part of the mean field is obtained by using the dispersion relations:

The total dispersive correction:

$$
J_{\Delta V_{W}}(E)=\frac{2}{\pi}\left(E-E_{F}\right) \int_{E_{0}}^{\infty} \frac{J_{W}\left(E^{\prime}\right)}{\left(E^{\prime}-E_{F}\right)^{2}-\left(E-E_{F}\right)^{2}} d E^{\prime}
$$

The volume dispersive correction:

$$
J_{\Delta V_{W V}}(E)=\frac{2}{\pi}\left(E-E_{F}\right) \int_{E_{0}}^{\infty} \frac{J_{W_{V}}\left(E^{\prime}\right)}{\left(E^{\prime}-E_{F}\right)^{2}-\left(E-E_{F}\right)^{2}} d E^{\prime}
$$

So, the surface dispersive correction is:

$$
J_{\Delta V_{W S}}(E)=J_{\Delta V_{W}}(E)-J_{\Delta V_{W V}}(E)
$$

\subsection{Depths of the Dispersive Corrections of the Real Optical Potential}

Determining the continuous energy variation of the depths of the dispersive corrections of the real optical potential:

The volume dispersive correction:

$$
\Delta \mathrm{V}_{\mathrm{V}}(\mathrm{E})=J_{\Delta V_{W V}}(E) / \mathrm{g}_{\mathrm{wv}}, \mathrm{MeV}
$$

The surface dispersive correction:

$$
\Delta \mathrm{V}_{\mathrm{S}}(\mathrm{E})=J_{\Delta V_{W S}}(E) / \mathrm{g}_{\mathrm{ws}}, \mathrm{MeV}
$$

So, the total dispersion potential $\Delta \mathcal{V}(r, E)$ calculated from "(9)", at $r=0$.

\subsection{Depth of the Total Real Central Potential}

Determining the continuous energy variation of the depth of the total real central potential:

We determine the depth from "(8)", at $r=0$,

Assumption that the Hatree-Fock term has a Wood-Saxon radial shape with energy-independent geometrical parameters $\left(r_{H F}, a_{H F}\right)$ is given by

$$
\mathcal{V}_{H F}(r, E)=\mathcal{V}_{H F}(E) f\left(r, R_{H F}, a_{H F}\right)
$$

Where the depth $\mathcal{V}_{H F}(E)$ is given by the following parametrization:

$$
\mathcal{V}_{H F}(E)=\mathcal{V}_{H F}\left(\mathrm{E}_{\mathrm{F}}\right) \mathrm{e}^{\left[\alpha_{\mathrm{HF}}\left(\mathrm{E}-\mathrm{E}_{\mathrm{F}}\right) / \mathcal{V}_{\mathrm{HF}}\left(\mathrm{E}_{\mathrm{F}}\right)\right]} \mathrm{E} \geq \mathrm{E}_{\mathrm{F}}
$$

Where $\alpha_{\mathrm{HF}}$, the slope parameter, $R_{\mathrm{HF}}=r_{H F} A^{1 / 3}$, radius parameter, $\mathcal{V}_{H F}\left(\mathrm{E}_{\mathrm{F}}\right)$ is the depth at Fermi energy.

\subsection{Volume Integral per Nucleon of the Real Potential}

Determining the continuous energy variation of the volume integral per nucleon of the real potential:

The volume integral per nucleon of the real potential is given by:

$$
J_{V}(E)=J_{H F}(E)+J_{\Delta V_{W}}(E)
$$

Where $J_{H F}(E)$, the volume integral per nucleon of the Hartree-Fock that can be written as follows,

$$
\mathrm{J}_{\mathrm{HF}}(\mathrm{E})=\mathcal{V}_{H F}(E) * g_{H F}
$$

Where $g_{H F}$, is given by 


$$
g_{H F}=\frac{4 \pi}{3} \frac{R_{H F}^{3}}{A_{t}^{*} A_{p}}\left[1+\left(\frac{\pi a_{\mathrm{HF}}}{R_{\mathrm{HF}}}\right)^{2}\right]
$$

\subsection{Radius Parameter of the Total Real Central Potential}

Determining the continuous energy variation of the radius parameter of the Woods-Saxon approximation to the full potential.

We determine the radius parameter of the Woods-Saxon approximation to the full optical potential from the equation:

$$
R_{V}(E)^{3}+\left(\pi a_{V}\right)^{2} R_{V}(E)-\left(\frac{3}{4 \pi}\right) g_{V}(E) A_{t} A_{p}=0
$$

Where $a_{V}$, diffuseness parameter and $g_{V}(E)$, can be determined from the relation:

$$
g_{V}(E)=J_{V}(E) / \mathcal{V}(E)
$$

So, the radius parameter will be:

$$
r_{V}(E)=R_{V}(E) A^{-1 / 3}
$$

\subsection{Comparing with the Global Parameterizations of the Optical Model Potential}

After calculating the depths, the geometrical parameters and the volume integral per nucleon of the mean field components, we have compared them with global parameterizations of the optical potential whose calculations have been performed in the DOMACNIP program:

1. Becchetti and Greenlees [9], its coding in the program
$\mathrm{BG}$, for

$$
\mathrm{E} \leq 50 \mathrm{Mev}, Z_{\mathrm{t}}=(20-92), \mathrm{A}_{\mathrm{t}}=(40-238)
$$

2. Menet et al [10], its coding in the program $\mathrm{ME}$, for

$$
30 \leq \mathrm{E} \leq 60 \mathrm{Mev}, \mathrm{Z}_{\mathrm{t}}=(20-82), \mathrm{A}_{\mathrm{t}}=(40-208)
$$

3. Varner et al [11], its coding in the program $\mathrm{CH}$, for

$$
16 \leq \mathrm{E} \leq 65 \mathrm{Mev}, \mathrm{Z}_{\mathrm{t}}=(20-83), \mathrm{A}_{\mathrm{t}}=(40-209)
$$

4. Koning and Delaroche [2], its coding in the program $\mathrm{KD}$, for

$$
0.001 \leq \mathrm{E} \leq 200 \mathrm{Mev}, \mathrm{Z}_{\mathrm{t}}=(12-83), \mathrm{A}_{\mathrm{t}}=(24-209)
$$

While the values of a spin-orbit coupling term of the mean field in our calculations are:

$$
\mathrm{V}_{\mathrm{SO}}=\mathrm{V}_{\mathrm{SO}_{\mathrm{KD}}}, \mathrm{W}_{\mathrm{SO}}=0, \mathrm{r}_{\mathrm{SO}}=\mathrm{r}_{\mathrm{SO}_{\mathrm{KD}}}, \mathrm{a}_{\mathrm{SO}}=\mathrm{a}_{\mathrm{SO}_{\mathrm{KD}}}
$$

Also, for the value of coulomb radius parameter, $r_{C}=r_{C_{K D}}$

\section{Results and Discussion}

The results According to DOMACNIP program are summarized as follows:

\subsection{Input Parameters}

The values of the input parameters of the titanium element nucleus and its natural isotopes are showed in the (Table 1):

Table 1. The input values of the constant mean field parameters and the changed atomic mass numbers, relative abundance [12-15].

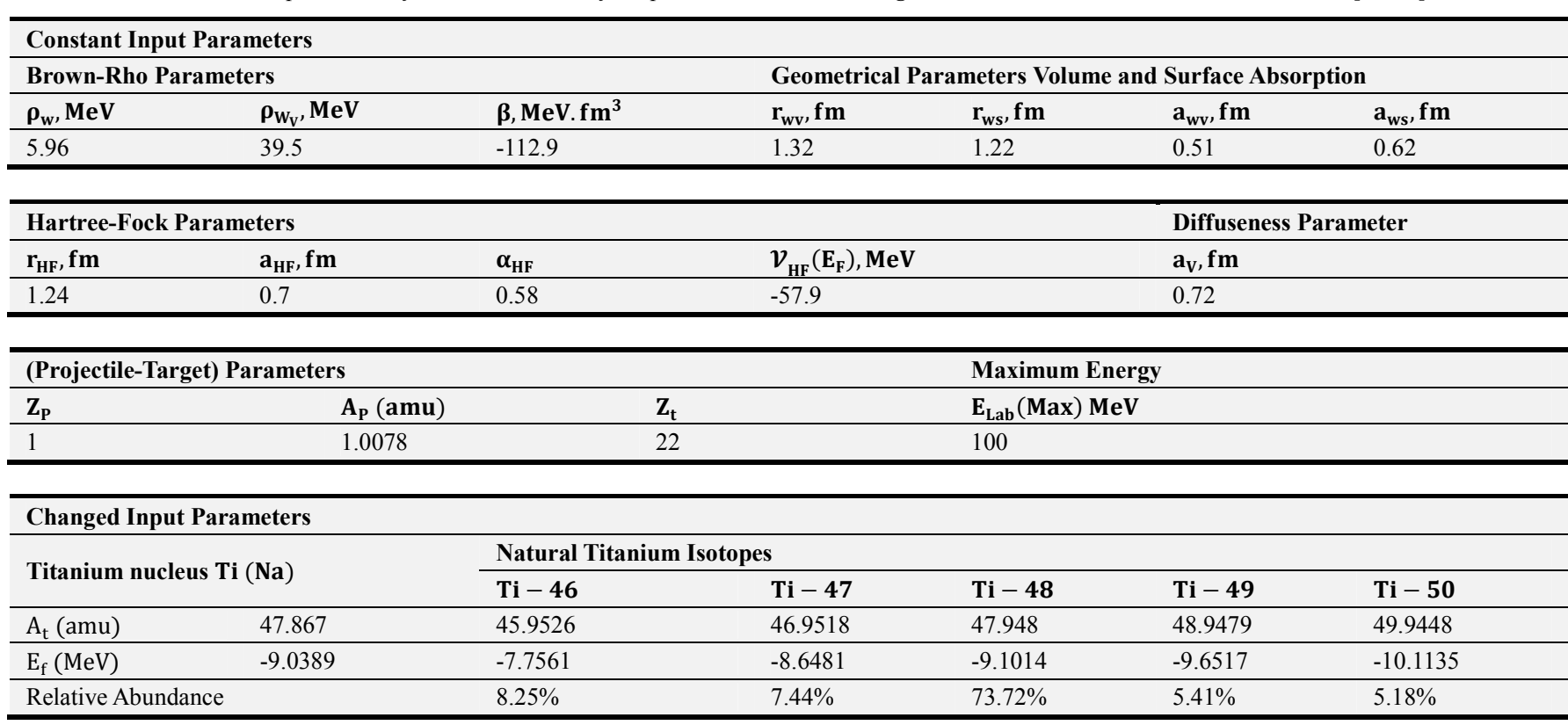

\subsection{Depths of the Mean Field}

The depths of the mean field are compared with these resulted from global parameterizations of the optical potential within the energy range $(1-100) \mathrm{MeV}$ and with calculation step of the previous range whose magnitude $1 \mathrm{MeV}$, as they are showed in the Figures 1-3. 

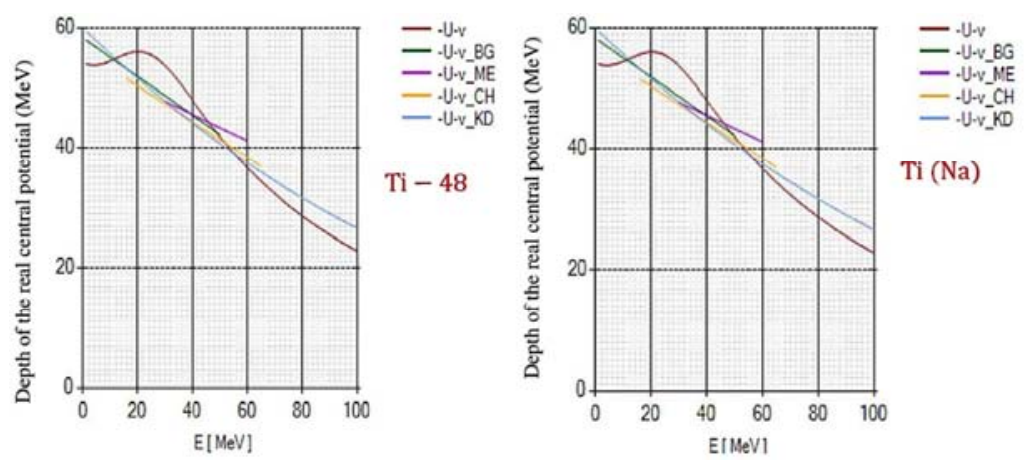

Figure 1. Depth of the real part of the mean field potential as a function of proton energy (the red line) compared with these resulted from global parametrization of the optical model potential.
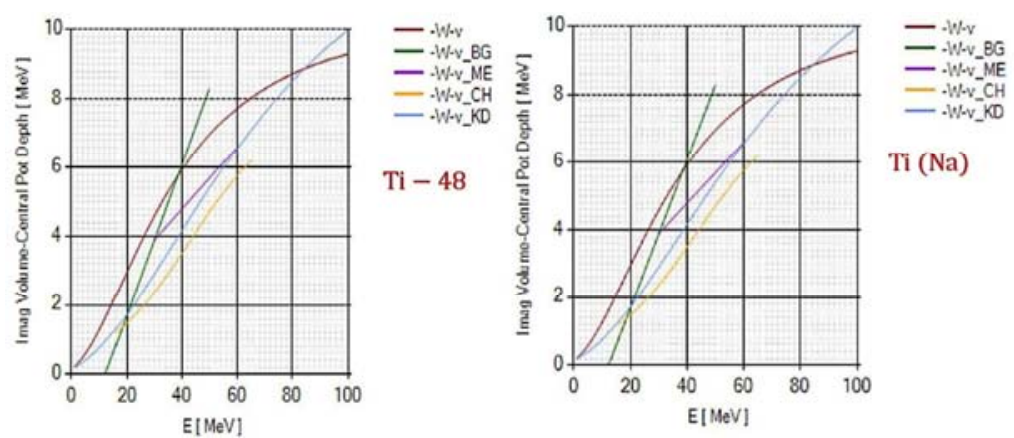

Figure 2. Depth of the volume component of the imaginary part of the mean field mean field potential as a function of proton energy (the red line) compared with these resulted from global parametrization of the optical model potential.
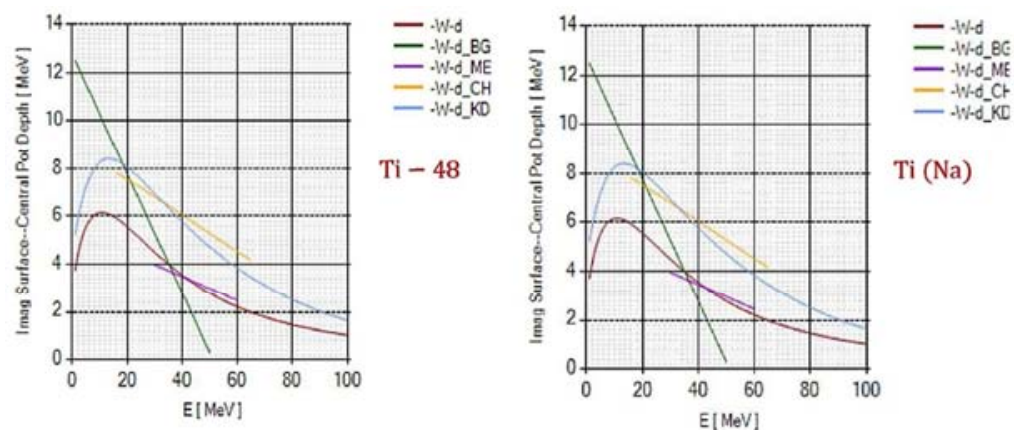

Figure 3. Depth of the surface-peaked component of the imaginary part of the mean field mean field potential as a function of proton energy (the red line) compared with these resulted from global parametrization of the optical model potential.

\subsection{The Real Radius Parameter of the Mean Field}

The real radius parameter of the Wood-Saxon approximation to the mean field potential with its HF approximation, within the energy range $\left(E_{f}-100\right) \mathrm{MeV}$ and with calculation step of the previous range whose magnitude $1 \mathrm{MeV}$, as it is showed in the Figure 4.
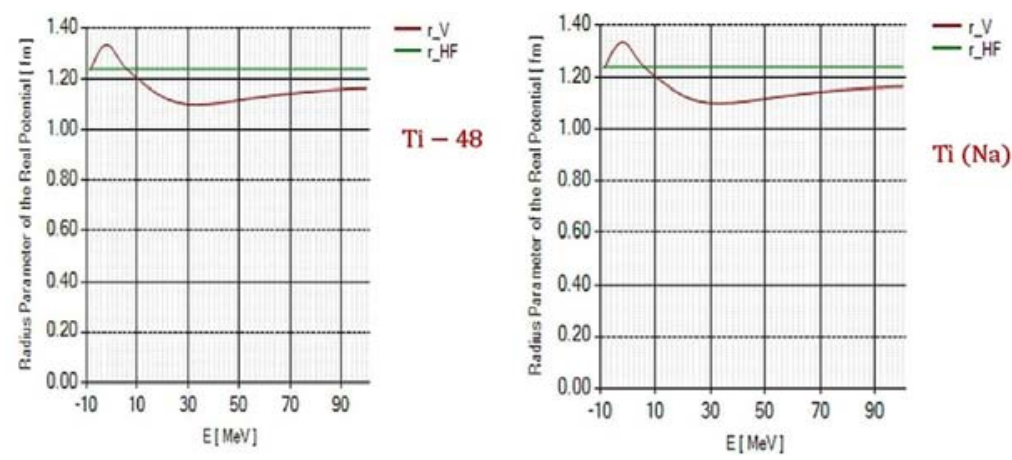

Figure 4. The energy dependence of the radius parameter of the Wood-Saxon approximation to the mean field potential with its HF approximation. 


\subsection{Total Reaction Cross Section}

The total reaction cross sections are compared with these resulted from global parameterizations of the optical potential and with available experimental data $[16,17]$, and are in

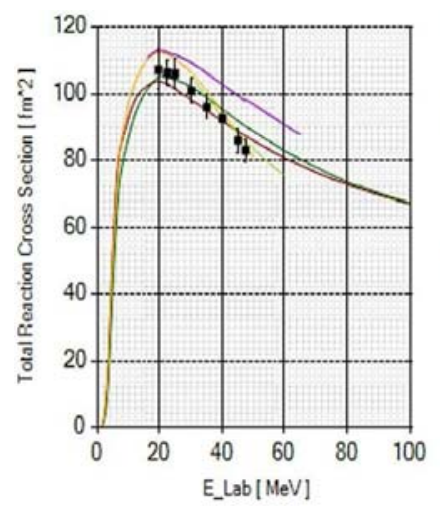

$\left(\mathrm{fm}^{2}=10 \mathrm{mb}\right)$, as it is showed in the Figure 5. There are excellent agreement with the experimental data and the global parametrization of the optical potential according to our calculations.

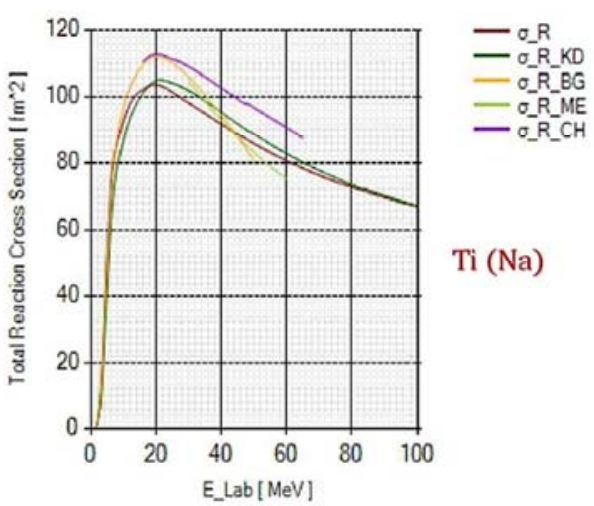

Figure 5. The energy dependence of the $\left(p+{ }^{(48, n a t u r a l)}\right.$ Ti) total cross section (the red line) compared with experimental values and with these resulted from global parametrization of the optical model potential.

\subsection{Differential Cross Sections and Polarization for Selected Energy}

The differential cross sections and polarization for selected energy compared with these resulted from global

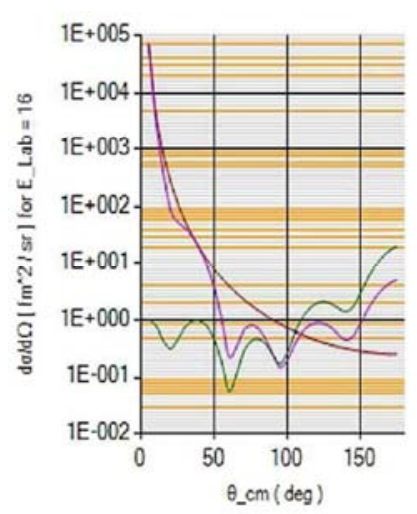

parameterizations of the optical potential, as which are showed in the Figures 6-9. There is an excellent agreement with the global parametrization of the optical model potential according to our calculations.

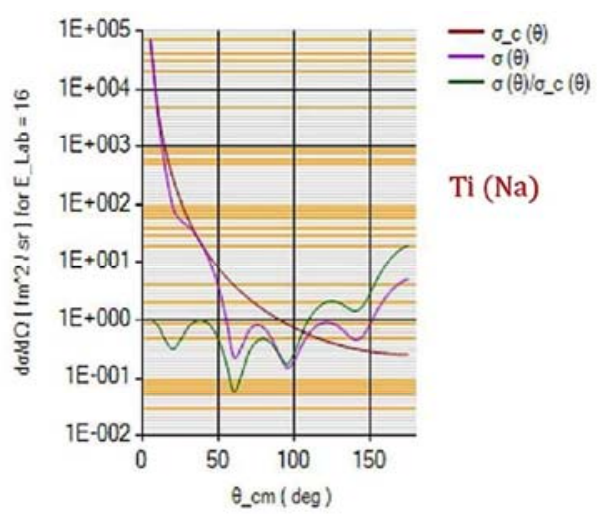

Figure 6. Dependence of the $\left(p+{ }^{(48, n a t u r a l)}\right.$ Ti) Coulomb, elastic differential cross sections and $\left(\sigma_{C} / \sigma\right)$ ratio upon the center-of-mass scattering angle, for $E_{\text {Lab }}=16 \mathrm{MeV}$.
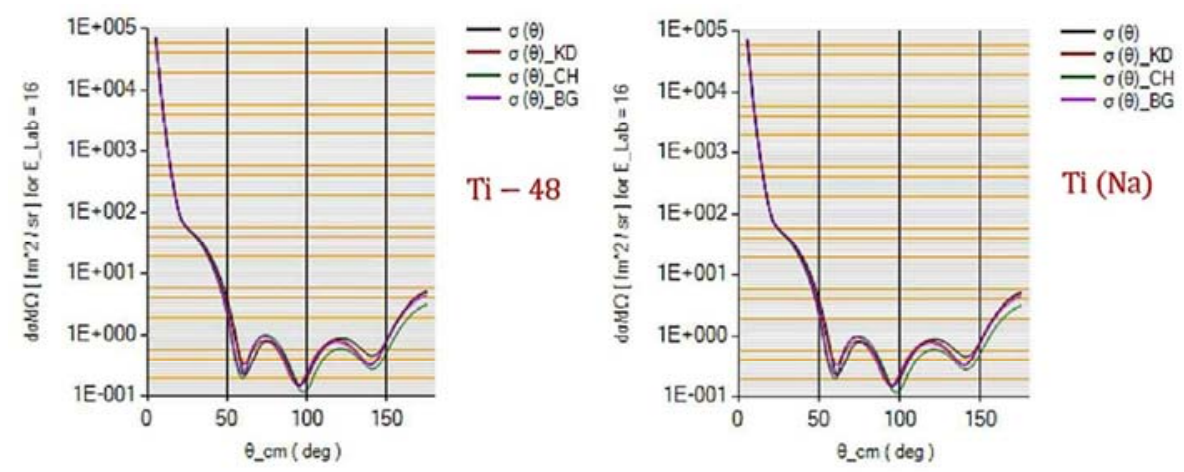

Figure 7. Dependence of the $\left(p+{ }^{(48, n a t u r a l)}\right.$ Ti) elastic differential cross section upon the center-of-mass scattering angle (the black line) compared with these resulted from global parametrization of the optical model potential, for $E_{L a b}=16 \mathrm{MeV}$. 

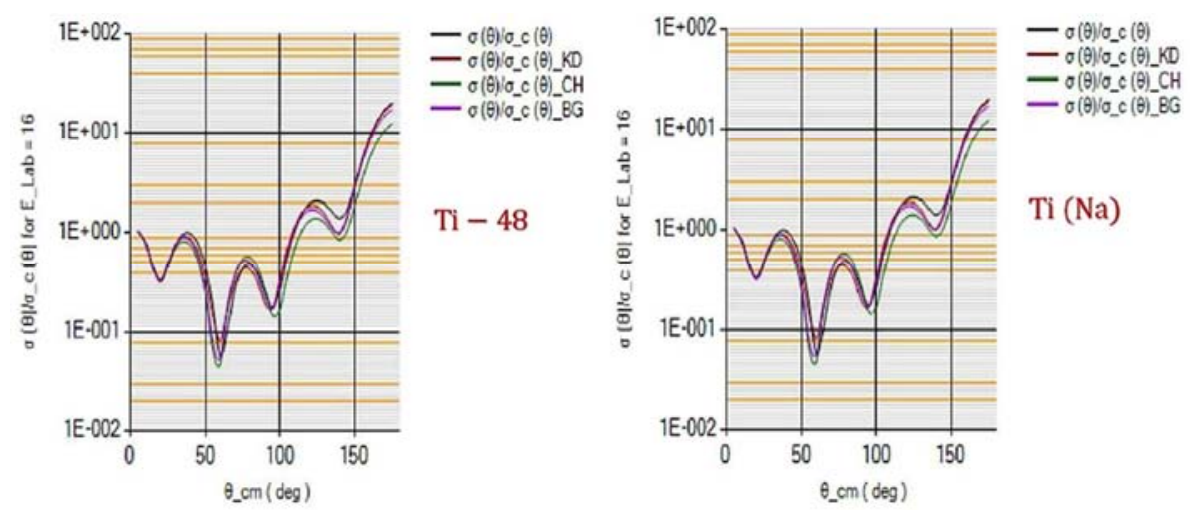

Figure 8. Dependence of the $\left(p+{ }^{(48, n a t u r a l)} T i\right)\left(\sigma_{C} / \sigma\right)$ ratio upon the center-of-mass scattering angle (the black line) compared with these resulted from global parametrization of the optical model potential, for $E_{L a b}=16 \mathrm{MeV}$.
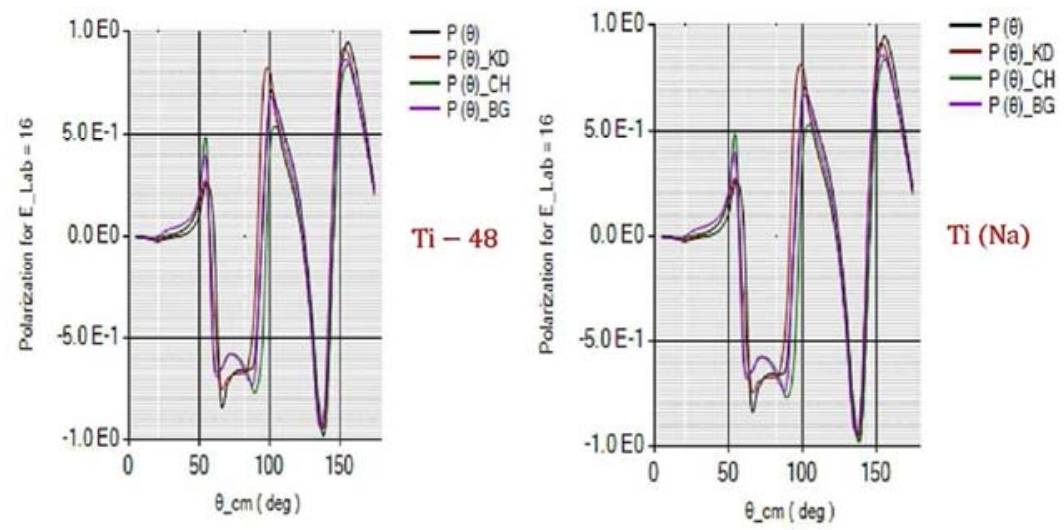

Figure 9. Dependence of the $\left(p+{ }^{(48, n a t u r a l)}\right.$ Ti) polarization upon the center-of-mass scattering angle (the black line) compared with these resulted from global parametrization of the optical model potential, for $E_{L a b}=16 \mathrm{MeV}$.

\section{Conclusion}

The important conclusions can be shown as follows:

i. Our result according to the dispersive optical model analysis of the proton scattering by titanium element nucleus and its natural isotopes have been drawn for constant input value of the mean field parameters using DOMACNIP program that has been designed for that purpose.

ii. Our calculation within the energy range (1$100) \mathrm{MeV}$ and with calculation step of the pervious range whose magnitude $1 \mathrm{MeV}$ of the continuous energy variation of the depths of the real and imaginary parts of the mean field were compared with these resulting from global parameterization of the optical model potential. In addition to continuous energy variation of the real radius parameter of the Wood-Saxon approximation to the mean field potential with its Hatree-Fock approximation of the nonlocal potential within the energy range $\left(E_{f}-100\right) \mathrm{MeV}$.

iii. Our prediction of the total reaction cross section data within the energy range $(1-100) \mathrm{MeV}$ and differential cross sections, polarization data for selected energy showed excellent agreement with available experimental data and with these resulted from global parameterization of the optical model potential and thus more reliable for calculation the cross sections of unknown interactions of elements nuclei and their isotopes.

\section{References}

[1] Hodgson, P. E. (1990). The unification of the nuclear optical potential, Contemporary Physics, 31: 5, 295-308, DOI: 10.1080/00107519008213780.

[2] Koning, A. J., \& Delaroche, J. P. (2003). Nucl. Phys. A713, 231.

[3] Mahaux, C., \& Sartor, R. (1991). Dispersion Relation Approach to the Mean Field and Spectral Functions of Nucleons in 40Ca, Nuclear Physics, A528, pp. 253-297, Elsevier Science Publishers B. V. (North-Holland).

[4] Mahaux, C., \& Satchler, G. R. (1993). Temporal Nonlocality of Nuclear and Atomic Mean Fields, Nuclear Physics, A560, pp. 5-22, Elsevier Science Publishers B. V. (North-Holland).

[5] IAEA, (2006). Handbook for Calculations of Nuclear Reaction Data, RIPL-2, IAEA in Austria, (Final report of a coordinated research project, IAEA-TECDOC-1506), pp. 4769.

[6] Melkanoff, M. A, Saxon, D. S, Jnodvik, J. S., \& Cantor, D. G. (1961). A Fortran Program for Elastic Scattering Analyses with the Nuclear Optical Model, University of California Press Berkeley and Los Angeles, Retrieved August 24, 2009 [EBook \#29784], online at www.gutenberg.org, p. 111. 
[7] Belal. A., \& Al-Mustafa, H. (2019). Program Design for Analyzing the Optical Model of the (Coulomb - Nuclear) Interference Potential, Journal of AL Baath University, HomsSyria, 41 (18), 71-102.

[8] Belal. A., \& Al-Mustafa, H. (2019). Program Design for Analyzing the Dispersive Optical Model of the (Coulomb Nuclear) Interference Potential, Journal of AL Baath University, Homs- Syria, 41 (17), 51-80.

[9] Bechetti, F. D., \& Greenlees, G. W. (1969)- Nucleon-Nucleus Optical Model Potential, Phys. Rev, 182, 1190P.

[10] Menet, J. H, Gross, E, Malanify, J., \& ZUCKER, A. (1972). Phys. Rev. C4, 1114 P.

[11] Varner, R. L, Thompson. W. J, Mcabee, T. L, Ludwig, E. J., \& Clegg, T. B. (1991)- A Global Nucleon Optical Model Potential. PHYSICS REPORTS (Review Section of Physics Letters) 201, NO. 2, pp. 57-119. Elsevier Science Publishers B. V. (North-Holland).

[12] Audi, G., \& Wapstra, A. H. (1993). The Isotopic Mass Data. Nucl. Phys A. 565, 1-65.

[13] Audi, G., \& Wapstra, A. H. (1995). The Isotopic Mass Data. Nucl. Phys A. 595, 409-480.
[14] Rosman, K. J. R., \& Taylor, P. D. P. (1999). The Percent Natural Abundance Data. (1997 report of the IUPAC Subcommittee for Isotopic Abundance Measurements). Pure Appl. Chem., 71, 1593-1607.

[15] Wieser, M. E. (2006). Atomic Weights of the Elements 2005. Department of Physics and Astronomy, University of Calgary, Calgary, Canada. (2006 IUPAC TECHNICAL REPORT). Pure Appl. Chem., Vol. 78, No. 11, pp. 2051-2066. DOI: 10.1351/pac200678112051.

[16] Nasr, T. N, Sourkes, A. M, Margaziotis, D. J., \& Cox, A. J (1978). Measurements of the Total Reaction Cross Section for Protons on $\mathrm{Ti}$ and $\mathrm{B}$ Between 20 and $50 \mathrm{MeV}$. Canadian Journal of Physical, volume 56, Page 56. (JANIS 4.0- LocalIncident proton data / EXFOR / Ti48 / (NON) / 00746.002, (8pts)).

[17] Menet, J. J, Groos, E. E, Malanify, J. J., \& Zucker, A. (1971). Total- Reaction- Cross Section Measurements for 30-60 MeV Protons and The Imaginary Optical Potential. Physical Review, Part C, Nuclear Physics, volume 4, Page 1114 (JANIS 4.0- Local- Incident proton data / EXFOR / Ti50 / (. NON) / 00081.018, (1pts)). 